\title{
Direct evidence for ferroelectric polar distortion in ultrathin lead titanate perovskite films
}

\author{
L. Despont,* C. Koitzsch, F. Clerc, M. G. Garnier, and P. Aebi \\ Institut de Physique, Université de Neuchâtel, CH-2000 Neuchâtel, Switzerland \\ C. Lichtensteiger and J.-M. Triscone \\ DPMC, Université de Genève, 24 Quai Ernest-Ansermet, CH-1211 Genève 4, Switzerland \\ F. J. Garcia de Abajo \\ Centro Mixto CSIC-UPV/EHU, 20080 San Sebastián, Spain \\ E. Bousquet and $\mathrm{Ph}$. Ghosez \\ Département de Physique, Université de Liège, B-4000 Sart-Tilman, Belgium \\ (Received 4 November 2005; published 10 March 2006)
}

\begin{abstract}
X-ray photoelectron diffraction is used to directly probe the intracell polar atomic distortion and tetragonality associated with ferroelectricity in ultrathin epitaxial $\mathrm{PbTiO}_{3}$ films. Our measurements, combined with $a b$ initio calculations, unambiguously demonstrate noncentrosymmetry in films a few unit cells thick, imply that films as thin as three unit cells still preserve a ferroelectric polar distortion, and also show that there is no thick paraelectric dead layer at the surface.
\end{abstract}

DOI: 10.1103/PhysRevB.73.094110

\section{INTRODUCTION}

Theoretical developments and experiments in the area of ferroelectrics have rapidly evolved over the last ten years, allowing further progress in the understanding of this remarkable phenomenon. In particular, "nanoscale" ferroelectrics have attracted considerable attention. ${ }^{1-5}$ The question of the existence of a critical thickness, in other words whether or not ferroelectricity can be maintained at reduced dimensions, is among the most exciting topics of the field today, with very active experimental ${ }^{6-9}$ and theoretical efforts. ${ }^{10-13}$

Probing ferroelectricity in thin films and nanostructures is a difficult task, which requires advanced techniques. Among these, scanning probe characterization based on piezoelectric microscopy has allowed a ferroelectric ground state to be identified down to $40 \AA$ in thin $\mathrm{Pb}\left(\mathrm{Zr}_{0.2} \mathrm{Ti}_{0.8}\right) \mathrm{O}_{3}$ films, ${ }^{6}$ and x-ray studies on $\mathrm{PbTiO}_{3}$ films suggested that $28 \AA$ films are ferroelectric. ${ }^{9}$ Dielectric and pyroelectric response measurements have allowed ferroelectricity to be identified in polymer films down to $10 \AA$ (two unit cells). ${ }^{14}$ More recently, ultrahigh vacuum scanning probe characterization based on electrostatic force microscopy was used to study ferroelectricity in barium titanate nanowires with diameters as small as $10 \mathrm{~nm} .{ }^{15}$ On insulating substrates, lateral periodicity was observed via x-ray diffraction in thin $\mathrm{PbTiO}_{3}$ films down to $12 \AA$ and attributed to alternately polarized domains. ${ }^{7,8}$ In all these studies, however, properties averaged over the complete ferroelectric structure were measured and no local information on the atomic displacements was obtained.

In contrast, the photoemission-based photoelectron diffraction (XPD) used in our study presents two interesting characteristics: it is naturally surface sensitive, due to an electron escape depth of $\sim 20 \AA$ (at the energy used here); and atomic displacements within the unit cell can be directly probed, allowing the noncentrosymmetric and tetragonal nature of the crystal lattice to be directly demonstrated. This turns out to be crucial for studying ultrathin films and for
PACS number(s): 77.55.+f, 77.80.-e, 61.14.Qp, 31.15.Ar

discriminating the behavior of the surface from that of the body of the film.

The paper is organized as follows. In Sec. II, we characterize the ferroelectric distortion of $\mathrm{PbTiO}_{3}$ and contrast it to the natural atomic relaxation appearing at surface and interface. In Sec. III A, we discuss aspects of the measurement methods while, in Sec. III B we show the noncentrosymmetry of a $20 \AA$ thick film using XPD. The Sec. III C is dedicated to the tetragonality measurements as a function of film thickness with XPD using $\mathrm{Pb}$ as emitter, down to $4 \AA$ (one unit cell). We summarize and conclude in Sec. IV.

\section{FERROELECTRIC DISTORTION VERSUS SURFACE RELAXATION}

The studies were carried out on $c$-axis-oriented perovskite $\mathrm{PbTiO}_{3}$ ultrathin films epitaxially grown on conducting $\mathrm{Nb}$ $-\mathrm{SrTiO}_{3}$ substrates. Above $490{ }^{\circ} \mathrm{C}$, bulk $\mathrm{PbTiO}_{3}$ is a paraelectric insulator and a simple cubic perovskite structure with a lattice parameter of $3.96 \AA$ ["para"-state, see Fig. 1(a)]. In this structure, the $\mathrm{Ti}$ and $\mathrm{O}$ atoms are in perfectly centrosymmetric positions with respect to the surrounding $\mathrm{Pb}$ cage. At lower temperature, the material becomes tetragonal and ferroelectric with $a$ - and $c$-axis parameters of 3.90 and $4.17 \AA$, respectively, ${ }^{16,17}$ as illustrated in Fig. 1(b). The ferroelectric phase is characterized by a noncentrosymmetric structure where the $\mathrm{O}$ and $\mathrm{Ti}$ atoms are unequally shifted with respect to $\mathrm{Pb}$. In a unit cell with the polar $c$-axis along the $z$ direction, $\mathrm{O}$ and Ti move either upward or downward (with a larger $\mathrm{O}$ displacement) resulting, respectively, in a down- and up-polarized state, as drawn in Fig. 1(b).

In the surface region (five top unit cells) that is probed by the XPD technique, the evidence of such a polar atomic distortion could be the signature of a ferroelectric up-or down - state but may also arise from the natural atomic relaxation at the film surface and interface already present in the 
(a) "Para"

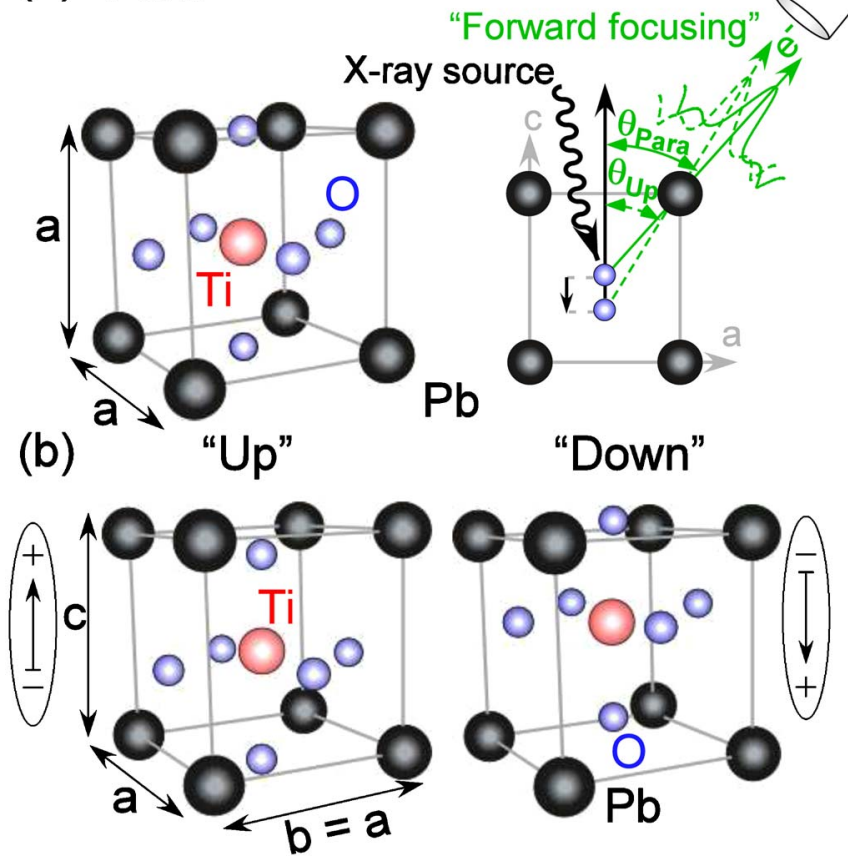

FIG. 1. (Color online) (a) Schematic of the measurement setup. Core electrons have well-defined binding energies, and their photoemission spectra exhibit characteristic emission lines. By selecting a particular emission line, photoelectrons from a given emitter can be chosen, thus probing the local real-space environment of the emitter. The interpretation is facilitated by forward focusing of electron flux along the emitter-scatterer direction. The difference between two "forward-focusing" peak positions is directly related to a modified emitter-scatterer direction, illustrated here by the paratoward up-state-O shift. Above $490{ }^{\circ} \mathrm{C}$, bulk $\mathrm{PbTiO}_{3}$ has a cubic unit cell, with the $\mathrm{O}$ and $\mathrm{Ti}$ atoms centered in the $\mathrm{Pb}$ cage (para -state). (b) Below $490{ }^{\circ} \mathrm{C}$, bulk $\mathrm{PbTiO}_{3}$ has a tetragonal unit cell: two equivalent ferroelectric configurations corresponding to two opposite polarizations, up-state and down-state. The displacements (in fractional units) from cubic phase sites are 0.111 and 0.037 for $\mathrm{O}$ and $\mathrm{Ti}$, respectively (Ref. 16).

paraelectric phase. A proper interpretation of our data therefore requires independent quantification of both effects. To that end, a reference configuration ("para-unrelaxed") is defined in Fig. 2(a); it corresponds to the truncated bulk paraelectric structure of $\mathrm{PbTiO}_{3}$ with the in-plane lattice constant constrained to that of $\operatorname{SrTiO}_{3}\left(a_{\mathrm{STO}}=3.90 \AA\right)$ and a consequent tetragonality $c_{0} / a_{\mathrm{STO}}=1.03$. ${ }^{9}$ Figure 2(b) ("ferrounrelaxed") shows the atomic distortion of the up state as determined for bulk tetragonal $\mathrm{PbTiO}_{3}$ by Nelmes and Kuhs ${ }^{16}$ with $c=4.17 \AA$. In order to quantify the surface relaxation, density-functional theory calculations ${ }^{18}$ were performed within the local-density approximation (LDA) using the ABINIT package. ${ }^{19}$ Two different supercells were considered: a thick $\mathrm{PbTiO}_{3}$ slab in vacuum [Fig. 2(c)] and a $\mathrm{SrTiO}_{3}$-(one unit cell) $\mathrm{PbTiO}_{3}$-vacuum stack ${ }^{20}$ [Fig. 2(d)]. Insulating $\mathrm{SrTiO}_{3}$ was considered in our simulations since $\mathrm{Nb}$ doping is not presently affordable at the first-principles level. ${ }^{21}$ To reproduce the substrate clamping effect, the inplane lattice constant was fixed to the relaxed $a$-axis value of bulk $\mathrm{SrTiO}_{3}{ }^{22}$ The atomic positions were then relaxed until the maximum residual atomic force was smaller than $40 \mathrm{meV} / \AA$. Our calculations were restricted to $(1 \times 1)$ surface periodicity and did not allow for an eventual antiferrodistortive (AFD) $c(2 \times 2)$ reconstruction. ${ }^{23-25}$ The latter is not excluded but, as discussed later, was not evidenced on our films at room temperature. The distortions in the upper half of each supercell are reported in Figs. 2(c) and 2(d) ("pararelaxed" state). For easy comparison to the experiment, because of the typical LDA underestimate of the lattice constant, ${ }^{22}$ the values are given as a percentage of $c_{0}$. The magnitudes of the ferroelectric and surface relaxation effects can now be compared. First, the cation-oxygen displacements due to ferroelectricity [11.6-8.3\% of $c_{0}$, Fig. 2(b)] are significantly larger than the displacements due to surface relaxation and rumpling [3.4-1.4\% of $c_{0}$, Fig. 2(c) and 3.3$1.5 \%$ of $c_{0}$, Fig. 2(d)]. Second, the mean layer displacement for the up state [Fig. 2(b)] and for the surface relaxation [Figs. 2(c) and 2(d)] are opposite. Third, the surface relaxation and rumpling effects are globally unaffected by the film thickness [Figs. 2(c) and 2(d)] and their amplitude decays very quickly in the interior of the film: they are already negligible two unit cells below the surface. This implies that the XPD measurements will be probing both the narrow relaxed surface region and a few unit cells below, essentially not affected by the surface relaxation.

\section{EXPERIMENTAL RESULTS AND DISCUSSION}

\section{A. Experimental details}

The samples used in this study are epitaxial, $c$-axis-oriented $\mathrm{PbTiO}_{3}$ thin films grown on conducting (001) $\mathrm{Nb}-\mathrm{SrTiO}_{3}$ substrates using off-axis radio-frequency magnetron sputtering. ${ }^{26,27}$ Topographic measurements using atomic force microscopy (AFM) showed that these films are essentially atomically smooth, with a root-mean-square roughness between 2 and $6 \AA$ over a $10 \times 10 \mu \mathrm{m}^{2}$ area. Room temperature $\mathrm{x}$-ray-diffraction measurements, for films with thickness $\geqslant 28 \AA$ allowed us to precisely determine the thickness and the $c$-axis parameter of the films, and to confirm their epitaxial "cube-on-cube" growth.

After growth and characterization, the films were transferred ex situ to a modified Vacuum Generators ESCALAB Mk II photoelectron spectrometer. The XPD measurement system comprises a hemispherical electron energy analyzer with a three-channel detector, an x-ray photon source with two possible energies $(h \nu=1253.6$ and $1740 \mathrm{eV}$ for $\operatorname{MgK} \alpha$ and $\operatorname{SiK} \alpha$ radiation, respectively), and a computer-controlled two-axis goniometer capable of rotating the photoelectron emission angle over the full hemisphere above the surface. ${ }^{28,29}$

The local geometry around a selected atom can be probed by performing an intensity vs emission-angle scan of a chosen photoemission line. Because of the chemical sensitivity of photoemission, a given atom type is then chosen by selecting one of its core levels. The outgoing photoemitted electrons exhibit a strongly anisotropic angular intensity distribution. This angular distribution is due to the interference of the directly emitted photoelectron wave with the scattered electron waves. The analysis of the interference (or diffrac- 


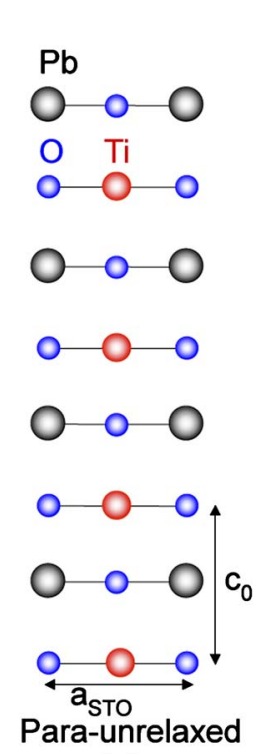

(a)

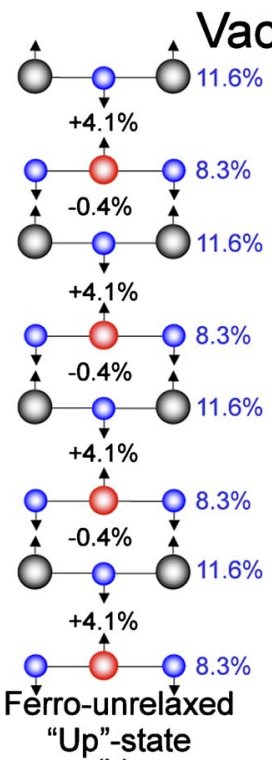

(b)

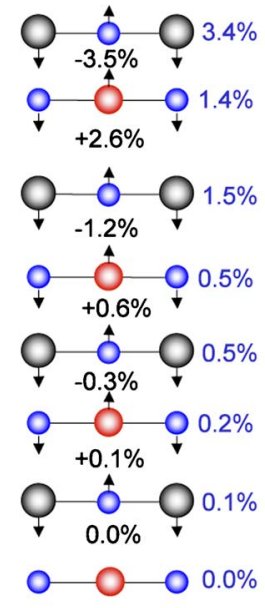

Para-relaxed

(c)

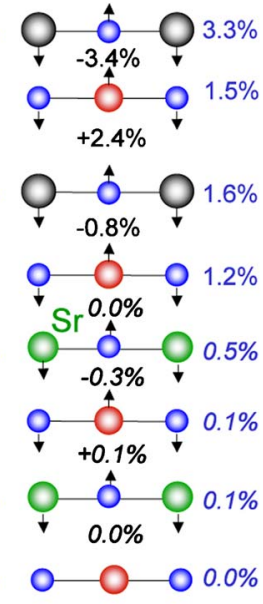

Para-relaxed

(d)

FIG. 2. (Color online) Schematic view of the atomic displacements resulting from ferroelectric distortion and from surface and interface relaxation in the uppermost layers of a $\mathrm{PbTiO}_{3}$ film epitaxially grown on $\mathrm{SrTiO}_{3}$. In bulk $\mathrm{PbTiO}_{3}$, epitaxial strain produces a tetragonality at zero polarization that can be estimated from the macroscopic elasticity theory as $c_{0} / a_{\mathrm{STO}}=1.03$. The ionic configuration resulting from the truncation of such a strained bulk paraelectric state (a) is considered as the reference structure. Freezing the bulk ferroelectric distortion (as reported in Ref. 16) into this reference structure results in the up-state shown in (b). Additionally, the natural ionic relaxation at the surface in the paraelectric state has been computed from first-principles (see text) both for a thick $\mathrm{PbTiO}_{3}$ slab in vacuum (c) and a $\mathrm{SrTiO}{ }_{3}-($ one unit cell) $\mathrm{PbTiO}_{3}$-vacuum stack (d). Numbers in black correspond to the change of interlayer distances. Numbers in gray (blue) corresponds to the atomic rumpling in each layer (cation-oxygen distance). All the values are in percent of $c_{0}$, except those in italic that concern $\mathrm{SrTiO}_{3}$ and are in percent of $a_{\mathrm{STO}}$.

tion) patterns is facilitated by the so-called forward-focusing effect taking place for photoelectron kinetic energies greater than $\approx 0.5 \mathrm{keV}$. When considering a row of atoms, scattering at the first few atoms along this row focuses the electron flux in the emitter-scatterer direction (for a review, see Ref. 30 and 31). This enhancement of the intensity in the emitterscatterer direction is schematically illustrated by the gray (green) curve in Fig. 1(a) (right part) for the centrosymmetric para-state (continuous line) and the up-state (dotted line). The forward-focusing effect is further amplified for electron scattering by heavy atoms. In a semiclassical picture, this can be understood as the focusing of the electron wave by the high number of protons in high atomic number atoms. ${ }^{30}$ Note that, despite the forward-focusing effect, the experimentally measured angles are sensitive to multiple interferences, refraction, and possible anisotropic atom vibrations at the surface. In the present case of $\mathrm{PbTiO}_{3}, \mathrm{~Pb}$ scattering is highly dominant compared to the scattering by other elements. ${ }^{32}$ As a first step, in order to probe the noncentrosymmetry, $\mathrm{O}$ was chosen as emitter-atom (O $1 s$ core level, $E_{k i n}=724.1 \mathrm{eV}$ ), since it has the largest displacement ${ }^{16}$ and has $\mathrm{Pb}$ scatterers as nearest neighbors (see Fig. 3). However, the $\mathrm{O}$ contribution from the $\mathrm{Nb}-\mathrm{SrTiO}_{3}$ substrate becomes non-negligible for films thinner than the photoelectron inelastic mean free path, making the study of films thinner than $20 \AA$ more difficult. As a second step, in order to probe the tetragonality of the films, i.e., the $c / a$ ratio of the $\mathrm{Pb}$ lattice (related to the polarization via the polarization-strain coupling as discussed below and, in detail, in Ref. 9), $\mathrm{Pb}$ was chosen as emitter atom ( $\mathrm{Pb} 4 f_{7 / 2}$ core level, $\left.E_{k i n}=1115.5 \mathrm{eV}\right)$, and $\mathrm{Pb}-\mathrm{Pb}$ forward-focusing directions were used. Since $\mathrm{Pb}$ atoms are absent from the substrate, this study can be done down to a monolayer of ferroelectric material.

\section{B. Noncentrosymmetric position of oxygen atoms}

First, considering oxygen as the emitter atom, fully automated computer code for calculating electron diffraction in atomic clusters (EDAC) via multiple scattering, ${ }^{33}$ based on the muffin-tin potential approximation ${ }^{34}$ was used to calculate the XPD pattern. Figure 3(a) shows four O $1 s$ core level emission $\left(E_{k i n}=724.1 \mathrm{eV}\right)$ interference patterns. One is the measurement made on a $20 \AA$ thin film while the three others are multiple scattering EDAC calculations of the up-, down-, and para-state. Intensities are plotted in a stereographic projection with the center corresponding to normal emission (polar angle $\theta=0^{\circ}$ ) and the outer border corresponding to grazing emission $\left(\theta=70^{\circ}\right)$. The strongest intensities (surrounded by white ellipses) correspond to the scattering of $\mathrm{O}$ $1 s$ photoelectrons by $\mathrm{Pb}$ nearest neighbors [see Fig. 3(b)]. The white circle is a guide to the eye indicating the polar angle of maximum intensity for the measured interference pattern. It is evident that the polar angle position of this peak, which is directly linked to the $\mathrm{O}-\mathrm{Pb}$ directions, is perfectly reproduced by the up-state calculation while the para- and the down-state simulations predict a different position. The up-state (downshifted $\mathrm{O}$ position) corresponds to a smaller polar emission angle $\left[\theta_{\text {up }}\right.$ in Fig. 3(b)] appearing closer to 


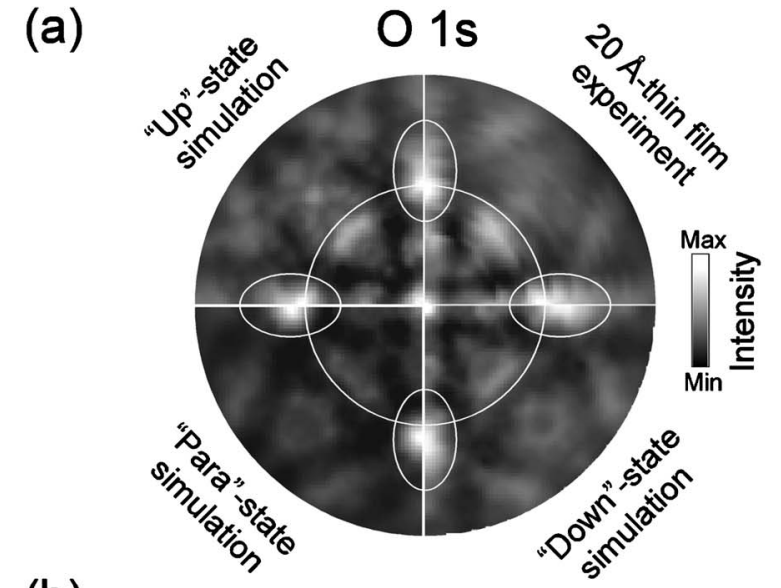

(b)

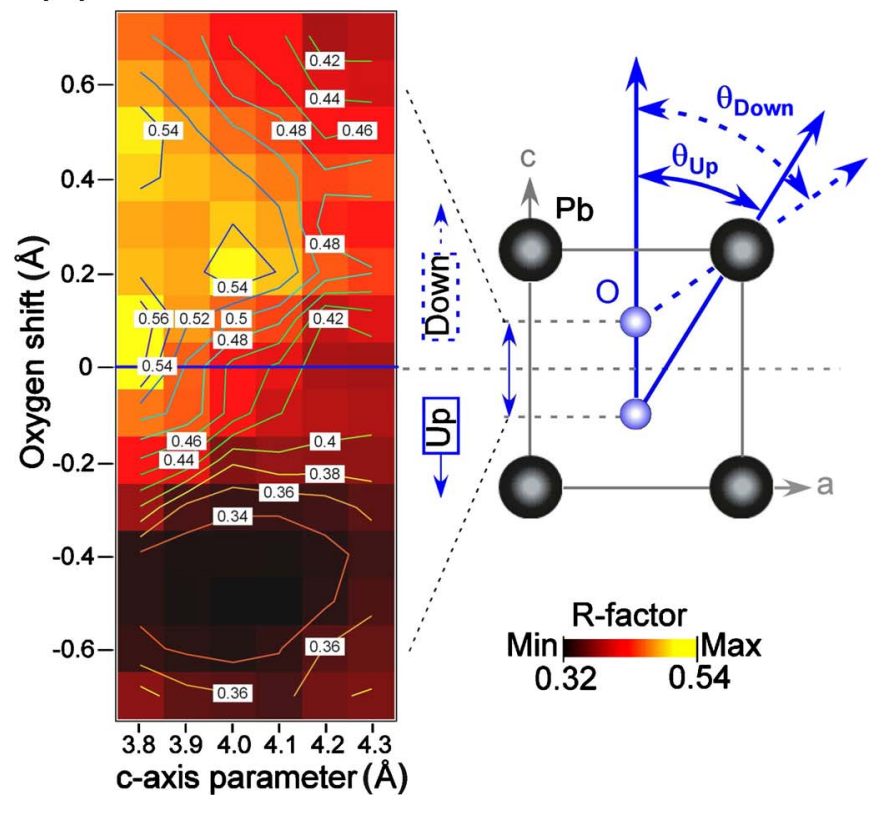

FIG. 3. (Color online) (a) Stereographic projection of experimental and theoretical $\mathrm{O} 1 \mathrm{~s}$ emission line intensities on quarter hemispheres for four different cases: experimental data for a $20 \AA$ thin film and theoretical simulations taking into account up-, para-, and down-state structures. Normal emission corresponds to the center of the plot and grazing emission $\left(\theta=70^{\circ}\right)$ to the outer border. (b) $R$-factor calculation to quantify the agreement between experiment and simulations for different structures, where a low $R$-factor corresponds to good agreement. A cut in the (100)-plane containing $\mathrm{Pb}$ and $\mathrm{O}$ atoms is shown to facilitate the discussion. A downward shift of $\mathrm{Pb}$ and $\mathrm{O}$ atoms optimizes the fit.

normal emission [center of the interference pattern in Fig. 3(a)]. Such measurements have also been performed on films with thicknesses of $\sim 500,200,100,60,44$, and $28 \AA$, and all perfectly reflect the characteristics of the up-state. ${ }^{36}$

These conclusions, drawn from visual inspection of the interference patterns locally around the intensity maximum [Fig. 3(a)], are confirmed by a global matching approach using a reliability $(R)$-factor to evaluate the quality of the fit between the complete experimental interference pattern data and theory [Fig. 3(b)]. The $c$-axis lattice-constant value and the $\mathrm{O}$ and Ti shifts are the adjustable structural parameters. A cut in the (100) plane, containing $\mathrm{Pb}$ and $\mathrm{O}$ atoms, is shown to facilitate the discussion. In the calculation, $\mathrm{O}$ and $\mathrm{Ti}$ atoms are moved together and the dipole is continuously changed from the down-state to the up-state, crossing over the para - state. The best fit corresponds to the minimal $R$-factor value, which is reached when $\mathrm{O}$ and $\mathrm{Ti}$ atoms are shifted below the centrosymmetric position [parameters used for the up-state simulation in Fig. 3(a)], with an $R$-factor value of $\approx 0.34$. In comparison, for the same $c$-axis parameter but the opposite $\mathrm{O}$ and $\mathrm{Ti}$ atom shifts [parameters used for the down-state simulation in Fig. 3(a)], the calculation gives a much higher $R$-factor of $\approx 0.47$. In between (zero O shift), in the centrosymmetric para-state, the $R$-factor is $\approx 0.45$ [parameters used for the para-state simulation in Fig. 3(a)].

It is important to note that surface relaxation and rumpling, neglected here, cannot weaken our conclusions; indeed, they would give a picture resembling a down-state, the corresponding $\mathrm{O}-\mathrm{Pb}$ atoms being shifted in the opposite direction than what is observed [see Fig. 2(c)]. Also, the possibility of a surface AFD reconstruction was explored without finding evidence for it in our room-temperature experiments.

This $R$-factor analysis, therefore, quantitatively confirms the observations made in Fig. 3(a); namely, that the measured interference pattern is best simulated with the up - state. This demonstrates unambiguously that, for a film as thin as $20 \AA$ the $\mathrm{O}$ atoms have a noncentrosymmetric position in the $\mathrm{Pb}$ cage corresponding to a nonvanishing spontaneous polarization, which is in agreement with the polar monodomain state observed in ultrathin $\mathrm{PbTiO}_{3}$ films with synchrotron x-ray scattering by Fong et al. ${ }^{35}$

Let us emphasize that piezoelectric AFM measurements performed after the XPD experiments on the thickest films $(\sim 500 \AA)$ confirmed the uniform up - state polarization while a uniform down-state polarization had been initially found for the same films just after growth. This confirms the monodomain character of the as-grown sample and also indicates that the films are uniformly switched from down- to up - state by exposure to our conventional x-ray source, attesting for the switchable character of the polarization. The details behind the switching are presently not known, but we believe that it occurs at the initial stage of the experiment while the measurement itself is essentially done in zero field. In fact, our results do not depend on the x-ray intensity, proving that the films are in equilibrium state during the measurements. ${ }^{37}$ As discussed below, the agreement between the tetragonality deduced from x-ray diffraction and XPD also suggests that the measurements are performed in similar conditions.

\section{Tetragonality via lead emission}

In a second step, considering $\mathrm{Pb}$ as the emitter atom, XPD was used to determine the tetragonality. As demonstrated in Ref. 9, below $200 \AA$ the tetragonality decreases as the film thickness decreases. This decrease is a consequence of the strong polarization-strain coupling in $\mathrm{PbTiO}_{3}$ and a signature of a reduced polarization in thin films. In Ref. 9, this polarization reduction was attributed to imperfect screening of the depolarizing field. ${ }^{12}$ With $\mathrm{XPD}$, using $\mathrm{Pb}$ as emitter, the tet- 


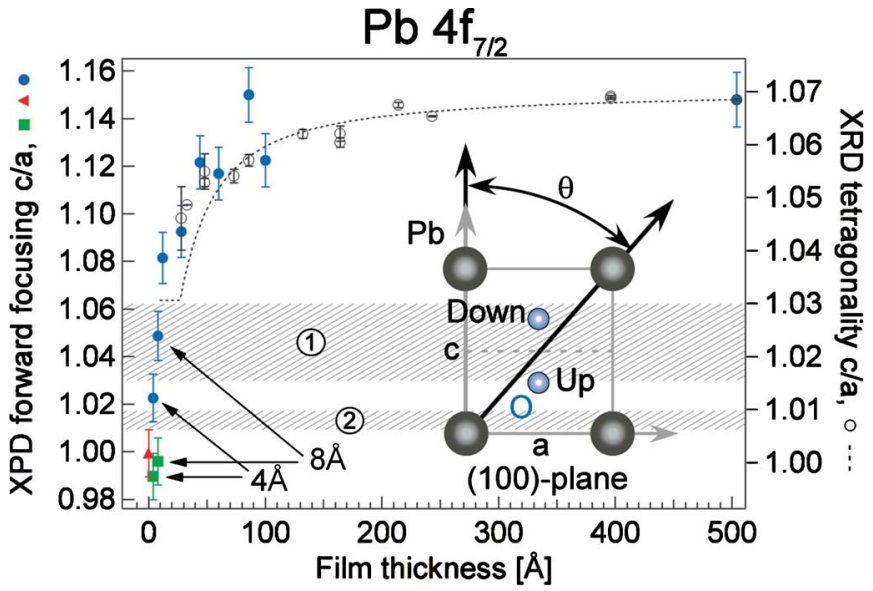

FIG. 4. (Color online) Tetragonality ( $c / a$ ratio) as a function of film thickness for $\mathrm{PbTiO}_{3}$ films (blue circles), for the $\mathrm{Nb}_{-} \mathrm{SrTiO}_{3}$ substrate through $\mathrm{PbTiO}_{3}$ films of one and two unit cells (green squares) and the $\mathrm{Nb}-\mathrm{SrTiO}_{3}$ substrate surface (red triangle). Black open circles are x-ray-diffraction data and the black dotted curve is the result from model Hamiltonian calculations from Ref. 9. Ribbons, labeled 1 and 2, indicate regions as discussed in the text. The left scale indicates the $c / a$ values extracted from the XPD experiment forward-focusing directions using $\theta$. The inset shows the crystallographic plane used to extract the $c / a$ ratio. $\mathrm{Pb}-\mathrm{Pb}$ scattering dominates compared to $\mathrm{Pb}-\mathrm{O}$ scattering allowing $\theta$ to be determined via $\mathrm{Pb}-\mathrm{Pb}$ forward focusing.

ragonality was measured down to the unit-cell level, as shown in Fig. 4. The absolute values of $c / a$, deduced from the forward-focusing angles, are particulary large. This might reflect a strong enhancement of the polarization in the probed surface region (of the order of $80 \%$ for $c / a=1.15$, from the $\mathrm{PbTiO}_{3}$ polarization-strain coupling), even larger than in the theoretical prediction of Ref. 10. However, as previously stated, we are not necessarily measuring the precise atomatom directions and the anomalously large forward-focusing $c / a$ might also be related to other effects (anisotropic atom vibrations at the surface, refraction, and multiple scattering interferences). Therefore, a comparison with x-ray diffraction (XRD) ${ }^{9}$ must be done at the relative level (Fig. 4, left and right scale).

To study the evolution of the tetragonality as a function of the film thickness, the measured XPD values are compared to the $c / a$ values obtained by XRD. The XPD measurement on Fig. 4 confirms the evolution of $c / a$ obtained from X-ray measurements in Ref. 9 and agrees with the theoretical prediction (dashed curve) relying on the suppression of polarization due to imperfect screening of the depolarizing field. ${ }^{9}$ The similar thickness dependence for the XPD (very surface sensitive) and the X-ray measurements (average on the whole film) implies that the polarization evolves at the surface in the same way as at the interior of the film and that there is no thick paraelectric dead layer at the surface. In addition, the XPD tetragonality measurement shows a continuous decrease of tetragonality down to the thickness of one unit cell. ${ }^{20}$ Two ribbons are drawn in Fig. 4, labeled 1 and 2. They indicate the regions within which $c / a$ values of 1.03 and 1.01 are crossed with respect to both $c / a$ scales. For film thicknesses above two unit cells, the $c / a$ values are larger than 1.03, the value expected at the bulk level for the paraelectric phase [resulting from the mechanical constraint imposed by the substrate, see also Fig. 2(a)]. This observation directly implies, via the polarization-strain coupling, that the films still have a finite-although progressively reduced-spontaneous polarization. At thicknesses of one or two unit cells, as can be seen in Fig. 4, c/a drops even more, reaching a value close to 1.01 for the one-unit-cell thick film. ${ }^{20}$ This further decrease highlights that macroscopic elasticity no longer applies at such thicknesses where the interlayer atomic distances are affected by surface relaxation and rumpling as shown by the $a b$ initio calculations [Fig. $2(d)]$. The measured tetragonality agrees with the computed value of 1.01 for the one-unit-cell thick relaxed paraelectric film, suggesting the absence of any additional ferroelectric distortion at this thickness.

\section{CONCLUSION}

This study thus directly demonstrates noncentrosymmetry, unambiguously a result of ferroelectricity in $\mathrm{PbTiO}_{3}$ thin films down to $20 \AA$. The measurements of the tetragonality, with a continuous decrease down to the bare substrate, show that even extremely thin films (three unit cells) have a $c / a$ value larger than 1.03, attesting for the presence of a nonvanishing spontaneous polarization at this thickness scale. As the film thickness is reduced to a single unit cell, the experiments, together with calculations, strongly suggest that both noncentrosymmetry and tetragonality are governed by surface effects, giving rise for our geometry to a polar-relaxed structure but without switchable ferroelectric distortion.

\section{ACKNOWLEDGMENTS}

We would like to thank M. A. Van Hove, C. Battaglia, and P. Baruch, for helpful discussions, and the whole Neuchâtel workshop and electric engineering team for efficient technical support. This project has been supported by the Swiss National Science Foundation through the National Center of Competence in Research "Materials with Novel Electronic Properties-MaNEP," the European Network of Excellence FAME, and the VolkswagenStiftung.
*Electronic address: Laurent.Despont@unine.ch; URL: http:// www.unine.ch/phys/spectro/homepage2.html

${ }^{1}$ C. H. Ahn, K. M. Rabe, and J.-M. Triscone, Science 303, 488 (2004).
${ }^{2}$ H. N. Lee, H. M. Christen, M. F. Chisholm, C. M. Rouleau, and

D. H. Lowndes, Nature (London) 433, 395 (2005).

${ }^{3}$ R. Waser and A. Rüdiger, Nat. Mater. 3, 81 (2004).

${ }^{4}$ M.-W. Chu, I. Szafraniak, R. Scholtz, C. Harnagea, D. Hesse, M. 
Alexe, and U. Gösele, Nat. Mater. 3, 87 (2004).

${ }^{5}$ L. B. I. I. Naumov and H. Fu, Nature (London) 432, 737 (2004).

${ }^{6}$ T. Tybell, C. H. Ahn, and J.-M. Triscone, Appl. Phys. Lett. 75, 856 (1999).

${ }^{7}$ S. K. Streiffer, J. A. Eastman, D. D. Fong, C. Thompson, A. Munkholm, M. V. Ramana Murty, O. Auciello, G. R. Bai, and G. B. Stephenson, Phys. Rev. Lett. 89, 067601 (2002).

${ }^{8}$ D. D. Fong, G. B. Stephenson, S. K. Streiffer, J. A. Eastman, O. Auciello, P. H. Fuoss, and C. Thompson, Science 304, 1650 (2004).

${ }^{9}$ C. Lichtensteiger, J.-M. Triscone, J. Junquera, and P. Ghosez, Phys. Rev. Lett. 94, 047603 (2005).

${ }^{10}$ P. Ghosez and K. M. Rabe, Appl. Phys. Lett. 76, 2767 (2000).

${ }^{11}$ B. Meyer and D. Vanderbilt, Phys. Rev. B 63, 205426 (2001).

${ }^{12}$ J. Junquera and P. Ghosez, Nature (London) 422, 506 (2003).

${ }^{13}$ N. Sai, A. M. Kolpak, and A. M. Rappe, Phys. Rev. B 72, 020101(R) (2005).

${ }^{14}$ A. Bune, V. M. Fridkin, S. Ducharme, L. M. Blinov, S. P. Palto, A. V. Sorokin, S. G. Yudin, and A. Zlatkin, Nature (London) 391, 874 (1998).

${ }^{15}$ W. S. Yun, J. J. Urban, Q. Gu, and H. Park, Nano Lett. 2, 447 (2002).

${ }^{16}$ R. J. Nelmes and W. F. Kuhs, Solid State Commun. 54, 721 (1985).

${ }^{17}$ F. Jona and G. Shirane, Ferroelectrics Crystals (Dover, New York, 1997).

${ }^{18}$ Teter norm conserving pseudopotentials were used with $\mathrm{Pb}$ $(5 d, 6 s, 6 p), \mathrm{Sr}(4 s, 4 p, 5 s)$, Ti $(3 s, 3 p, 3 d, 4 s)$, and $\mathrm{O}(2 s, 2 p)$ treated as valence states, a plane-wave energy cutoff of $35 \mathrm{Ha}$ and $6 \times 6 \times 1 k$-point sampling of the Brillouin zone.

${ }^{19}$ X. Gonze, J.-M. Beuken, R. Caracas, F. Detraux, M. Fuchs, G.-M. Rignanese, L. Sindic, M. Verstraete, G. Zerah, F. Jollet, M. Torrent, A. Roy, M. Mikami, Ph. Ghosez, J.-Y. Raty, and D. C. Allan, Comput. Mater. Sci. 25, 478 (2002).

${ }^{20}$ Note that a film with thickness of one unit cell corresponds to two $\mathrm{Pb}-\mathrm{O}$ and one Ti-O layers.

${ }^{21} \mathrm{The} \mathrm{Nb}$ doping should have a minor effect on the relaxation at the paraelectric level except for ultrathin films for which the metallic character of the surface could allow a larger polar relaxation of the $\mathrm{PbTiO}_{3}$ surface.

${ }^{22}$ At the bulk level, the calculations provide very similar theoretical in-plane lattice constant for cubic paraelectric $\mathrm{SrTiO}_{3}$ $(a=3.85 \AA)$ and tetragonal ferroelectric $\mathrm{PbTiO}_{3}(a=3.86 \AA$, $c=3.98 \AA$ ) in spite of a global underestimate of the experimental value $(a=3.90 \AA)$ typical of the LDA.

${ }^{23}$ C. Bungaro and K. M. Rabe, Phys. Rev. B 71, 035420 (2005).

${ }^{24}$ M. Sepliarsky, M. Stachiotti, and R. L. Migoni, cond-mat/ 0503524 (unpublished).

${ }^{25}$ A. Munkholm, S. K. Streiffer, M. V. Ramana Murty, J. A. Eastman, C. Thompson, O. Auciello, L. Thompson, J. F. Moore, and G. B. Stephenson, Phys. Rev. Lett. 88, 016101 (2002).

${ }^{26}$ C. B. Eom, J. Z. Sun, B. M. Lairson, S. K. Streiffer, A. F. Marshall, K. Yamamoto, S. M. Anlage, J. C. Bravman, T. H. Geballe, S. S. Laderman, R. C. Taber, and R. D. Jacowitz, Physica C 171, 354 (1990).

${ }^{27}$ C. Lichtensteiger and J.-M. Triscone, Integr. Ferroelectr. 61, 143 (2004).

${ }^{28}$ J. Osterwalder, T. Greber, A. Stuck, and L. Schlapbach, Phys. Rev. B 44, 13764 (1991).

${ }^{29}$ D. Naumović, A. Stuck, T. Greber, J. Osterwalder, and L. Schlapbach, Phys. Rev. B 47, 7462 (1993).

${ }^{30}$ W. F. Egelhoff, Jr., Crit. Rev. Solid State Mater. Sci. 16, 213 (1990).

${ }^{31}$ C. S. Fadley, Synchrotron Radiation Research: Advances in Surface Science (Plenum, New York, 1990).

${ }^{32}$ L. Despont, C. Lichtensteiger, F.Clerc, M. G. Garnier, F. J. Garcia de Abajo, M. A. Van Hove, J.-M. Triscone, and P. Aebi, Eur. Phys. J. B 49, 141-146 (2006).

${ }^{33}$ F. J. Garcia de Abajo, M. A. Van Hove, and C. S. Fadley, Phys. Rev. B 63, 075404 (2001).

${ }^{34}$ J. B. Pendry, Low Energy Electron Diffraction (Acamedic Press, London, 1974).

${ }^{35}$ D. D. Fong, C. Cionca, Y. Yacoby, G. B. Stephenson, J. A. Eastman, P. H. Fuoss, S. K. Streiffer, C. Thompson, R. Clarke, R. Pindak, and E. A. Stern, Phys. Rev. B 71, 144112 (2005).

${ }^{36}$ The presence of alternating $180^{\circ}$ domains in our films can be ruled out from the XPD measurement because the two characteristic up- and down-state forward-focusing peaks are not observed simultaneously in the experimental diffractogram, Fig. 3(a). This contrasts with the results of Fong et al. (Ref. 8) on insulating substrates.

${ }^{37}$ The influence of the $\mathrm{x}$-ray intensity on the tetragonality was measured by investigating different $\mathrm{x}$-ray powers. A modification of the tetragonality would have indicated, via the polarizationstrain coupling, a variation of the spontaneous polarization. However, no such modification was found. 